Gut and Liver, Vol. 10, No. 4, July 2016, pp. 549-555

\title{
A Comparative Randomized Trial on the Optimal Timing of Dexamethasone for Pain Relief after Endoscopic Submucosal Dissection for Early Gastric Neoplasm
}

\author{
Jeung Hui Pyo, Hyuk Lee, Yang Won Min, Byung-Hoon Min, Jun Haeng Lee, Poong-Lyul Rhee, and Jae J. Kim \\ Department of Medicine, Samsung Medical Center, Sungkyunkwan University School of Medicine, Seoul, Korea
}

Background/Aims: The aim of this study was to compare the clinical effects of preoperative and postoperative dexamethasone on pain after endoscopic submucosal dissection (ESD) for early gastric neoplasm. Methods: Forty patients with early gastric neoplasm who were scheduled for ESD were randomized into two groups according to the timing of steroid administration: preoperative ("pre", n=20) and postoperative ("post", $n=20$ ) steroid administration. The pre group received $0.15 \mathrm{mg} / \mathrm{kg}$ dexamethasone before ESD and placebo after, and the post group received pre-ESD placebo and post-ESD dexamethasone. The present pain intensity (PPI) index and the short-form McGill pain (SF-MP) questionnaire were evaluated. Results: The primary outcome was PPI score at 6 hours after ESD. There was a greater reduction in 6 -hour PPI in the pre group than in the post group $(2.1 \pm 0.8$ vs $3.0 \pm 1.1$, respectively; $p=0.006$ ). The immediate PPI was also significantly lower in the pre group than in the post group (1.6 \pm 0.6 vs $2.9 \pm 0.6$, respectively; $p<0.001$ ), and the total SF-MP scores were significantly lower in the pre group than in the post group both immediately and at 6 hours after the operation. Conclusions: Preoperative administration of dexamethasone may produce a superior analgesic effect in patients who undergo ESD compared with the postoperative administration of dexamethasone. (Gut Liver 2016;10:549555)

Key Words: Endoscopic submucosal dissection; Stomach neoplasms; Pain; Dexamethasone

\section{INTRODUCTION}

Endoscopic submucosal dissection (ESD) is accepted as an excellent treatment modality based on clinical evidence of the merits of the technique over the past decade, and it has been proposed as the gold standard treatment for early gastric cancer or premalignant lesions in the stomach. ${ }^{1}$ Although ESD is a less invasive procedure than surgical gastrectomy, it requires a high level of skill and careful observation for associated complications such as bleeding and perforation. Pain is a minor ESD complication, but also the most frequently reported. ${ }^{2}$ Post-ESD pain appears to be associated with ulcer defects and/or transmural burns or transmural air leaks, ${ }^{3,4}$ and inadequate pain control has health and socioeconomic consequences such as prolonged hospitalization and decreased patient satisfaction. However, these conditions have often been overlooked, and only a few published reports have studied management strategies for epigastric pain associated with ESD., ${ }^{2,5-7}$ Previous studies have reported the efficacy of local lidocaine injection, ${ }^{6}$ transdermal fentanyl, ${ }^{7}$ topical bupivacaine, and triamcinolone acetonide injection. ${ }^{2}$ However, these studies focused on regulating the pain, not preventing it.

Glucocorticoids have a variety of anti-inflammatory, immunosuppressive effects and also analgesic effects within the immediate postoperative period. $^{8-10}$ Recently, one meta-analysis showed that intermediate-dose dexamethasone is an effective and safe strategy for postoperative pain control. ${ }^{11}$ Our prior research indicated that single-dose postoperative intravenous dexamethasone after ESD effectively relieved epigastric pain. ${ }^{5}$ However, the optimal timing of administrating dexamethasone for effective analgesia of post-ESD pain has not been studied. The concept of preemptive analgesia developed as a consequence of numerous studies on reducing postoperative pain by introducing a preoperative analgesic regimen in order to reduce central sensitization of any nociceptive stimuli during the sur-

Correspondence to: Hyuk Lee

Division of Gastroenterology, Department of Medicine, Samsung Medical Center, Sungkyunkwan University School of Medicine, 81 Irwon-ro, Gangnam-gu, Seoul 06351, Korea

Tel: +82-2-3410-3283, Fax: +82-2-3410-6983, E-mail: leehyuk@skku.edu

Received on July 1, 2015. Revised on September 28, 2015. Accepted on October 23, 2015. Published online April 28, 2016 pISSN 1976-2283 eISSN 2005-1212 http://dx.doi.org/10.5009/gnl15302

() This is an Open Access article distributed under the terms of the Creative Commons Attribution Non-Commercial License (http://creativecommons.org/licenses/by-nc/4.0) which permits unrestricted non-commercial use, distribution, and reproduction in any medium, provided the original work is properly cited. 
gery. ${ }^{12-15}$ There are a number of studies on the use of perioperative glucocorticoids to reduce postoperative pain, and some reported statistically significant reduced pain. ${ }^{16-19}$

Therefore, this study investigated the hypothesis that dexamethasone is more effective when administered before rather than after ESD in relieving epigastric pain. We used pain scoring system categories in order to evaluate post-ESD epigastric pain.

\section{MATERIALS AND METHODS}

\section{Study participants}

Patients who were 20 years of age or older who were scheduled to undergo ESD for pathologically diagnosed gastric adenoma or cancer between July and October 2014 at Samsung Medical Center, Sungkyunkwan University School of Medicine, Seoul, Korea, were enrolled in this prospective, randomized, double-blinded, placebo-controlled study. Written informed consent was obtained from all patients or a responsible family member. Exclusion criteria were (1) administration of a painkiller on a regular basis or within 48 hours of enrollment; (2) confirmation of any other disease that could induce epigastric pain, such as peptic ulcer disease or gastroesophageal reflux disease; (3) presence of multiple gastric ESD lesions; (4) history of gastric surgery at enrollment; and (5) presence of a severe underlying disease, including infection, cardiopulmonary disease, and diabetes. The standard eligibility criteria for ESD were (1) histological diagnosis of a well- or moderately differentiated adenocarcinoma or dysplasia (adenoma); (2) tumor invasion of the mucosa or minute submucosal layers defined by endoscopic ultrasonography; (3) tumor size $\leq 3 \mathrm{~cm}$ if minute submucosal invasion or a tumor with ulceration was suspected; and (4) a tumor of any size if it was a differentiated adenocarcinoma without ulceration or submucosal invasion. Initially, 47 consecutive patients with the aforementioned characteristics were screened to determine their eligibility for the study. Seven of these patients were excluded because they did not provide informed consent $(n=4)$, had multiple lesions $(n=1)$, or were taking a nonsteroidal anti-inflammatory drug $(n=2)$. The remaining 40 patients were randomized into two groups: preoperative ("pre", $\mathrm{n}=20$ ) and postoperative ("post", $\mathrm{n}=20$ ) steroid administration. The pre group were given $0.15 \mathrm{mg} / \mathrm{kg}$ dexamethasone intravenously immediately before ESD and placebo (saline only) immediately after, whereas the post group received matching pre-ESD placebo and post-ESD dexamethasone.

The study was approved by the Independent Institutional Review Board of Samsung Medical Center and conformed to the ethical guidelines of the 1975 Helsinki Declaration. The study was registered with UMIN-CTR, identification number UMIN000016614.

\section{Study design}

Patients were randomly assigned in a one-to-one ratio to the pre or the post group according to a computer-generated randomization list, and the allocation was concealed from the researchers who assessed and enrolled the participants. To prevent bleeding from ESD-induced ulcer, all patients received an intravenous injection of the proton pump inhibitor pantoprazole (40 mg twice daily [bid]) for 2 consecutive days. From the third day following the ESD, oral lansoprazole ( $40 \mathrm{mg}$ ) was administered once a day to all patients for 28 days.

\section{ESD procedures}

All endoscopic procedures were performed as described in our previous report. ${ }^{20}$ Procedure time was defined as the time from circumferential marking around the lesion to the complete removal of the lesion. Complete resection was defined as negative tumor from the resection margin, and en bloc resection was defined as tumor resected in one piece. The tumor size was calculated with two vertical maximum diameters. Bleeding was defined as intraoperative massive bleeding that needed transfusion or postoperative bleeding that required endoscopic hemostasis. The degree of bleeding (minimal, moderate, and severe) and the degree of fibrosis (minimal, moderate, and severe) were evaluated during the procedures.

\section{Primary and secondary outcome measurements}

To evaluate epigastric pain after ESD, the patients completed a questionnaire about the degree of abdominal pain using the present pain intensity (PPI) scale and the short-form McGill pain (SF-MP) questionnaire immediately and at 6, 12, and 24 hours postoperative. The PPI scale rates pain intensity on a 5-point scale ( $0=$ no pain, $5=$ pain as bad as it could be). ${ }^{21}$ The SF-MP questionnaire consists of 15 descriptors (11 sensory, 4 affective) that are rated on a 3 -point intensity scale $(0=$ none, $1=$ mild, $2=$ moderate, or $3=$ severe). The SF-MP score also includes the PPI and a visual analog scale. Total SF-MP scores range from 0 to 60 , with a higher score indicating worse pain. ${ }^{21}$ A single trained research assistant measured and recorded the pain scores. Because maximum pain was observed in patients at 6 hours after ESD in our pilot study, PPI score at 6 hours after ESD was chosen as the primary outcome. The secondary outcomes were PPI scores at 0, 12, and 24 hours, SF-MG pain scores, the need for an additional painkiller, and the presence of acute or delayed bleeding complications. Patients with epigastric pain after ESD were given pethidine $\mathrm{HCl}$ (Pethidine $\mathrm{HCl}$ Injection 25 mg/vial; Hana Pharm., Seoul, Korea) once intramuscularly as an additional painkiller. If the patient suffered from sustained pain after the first pethidine injection, it was given once more, regardless of time. Other data collected were age, sex, body mass index (BMI), tumor size, tumor location, degree of bleeding, and degree of fibrosis. These variables were 
compared between the two groups.

\section{Statistical analysis}

The sample size was calculated on the basis of our previously published results. ${ }^{5}$ The earlier study on dexamethasone for pain relief after ESD was performed with 20 patients per group and showed 6-hour PPI scores of 1.67 (standard deviation [SD], 1.03) for the dexamethasone group and 2.67 (SD, 1.03) for the placebo group. Based on a power of $80 \%$ and a two-tailed $\alpha$ of 0.05 , the sample size required for the present study was determined to be 18 per group, for a total of 36 patients. The final sample size required was 40 patients to accommodate a dropout rate of $10 \%$.

The data for all variables with normal distributions are expressed as mean \pm SD. Comparisons between variables were analyzed by t-test. Categorical variables were expressed as percentages and compared using the chi-square test or Fisher exact test. Binary logistic regression tests were used for multivariate analysis. Characteristics with a univariate significance of $\mathrm{p}<0.05$ were candidates for multivariate analysis. Repeated-measure variables such as total SF-MP score, sensory SF-MP score, affective SF-MP score, and PPI were analyzed using a linear mixed model and generalized estimating equations for numerical and categorical measures, respectively, with fixed and random effects. When the interactions of group, time, and group by time with the other variables showed statistical significance, post hoc analysis was carried out with Bonferroni correction to adjust for multiple comparisons. All probabilities were two-tailed, and the level of statistical significance was defined as $p<0.05$. Statistical analysis was performed using SPSS version 20.0 (IBM Corp., Armonk, NY, USA).

\section{RESULTS}

\section{Patients and tumor characteristics}

The 20 patients who were randomized into the pre group received $0.15 \mathrm{mg} / \mathrm{kg}$ dexamethasone intravenously before ESD and placebo after, whereas the 20 patients who were randomized into the post group received matching pre-ESD placebo and post-ESD dexamethasone. There were no dropouts among the patients; all 40 were enrolled in the study (Fig. 1). There were no significant differences between the two groups in baseline characteristics (Table 1). All patients except one in the post group underwent complete or en bloc resections.

Table 1. Baseline Characteristics

\begin{tabular}{|c|c|c|c|}
\hline Characteristic & $\begin{array}{l}\text { Pre group } \\
(n=20)\end{array}$ & $\begin{array}{l}\text { Post group } \\
\quad(n=20)\end{array}$ & p-value \\
\hline Age, yr & $55.1 \pm 8.2$ & $52.3 \pm 9.1$ & 0.305 \\
\hline Male sex & $12(60.0)$ & $11(55.0)$ & 0.999 \\
\hline BMI, kg/m² & $22.3 \pm 1.9$ & $22.2 \pm 1.9$ & 0.867 \\
\hline Size, $\mathrm{mm}$ & $1,669.9 \pm 456.3$ & $1,767.6 \pm 534.7$ & 0.538 \\
\hline Location & & & 0.339 \\
\hline Upper & $6(30.0)$ & $5(25.0)$ & \\
\hline Middle & $3(15.0)$ & $7(35.0)$ & \\
\hline Lower & $11(55.0)$ & $8(40.0)$ & \\
\hline Procedure time, min & $45.4 \pm 11.9$ & $41.6 \pm 11.7$ & 0.309 \\
\hline Bleeding & & & 0.539 \\
\hline Minimal & $11(55.0)$ & $12(60.0)$ & \\
\hline Moderate & $9(45.0)$ & $8(40.0)$ & \\
\hline Severe & 0 & 0 & \\
\hline Fibrosis & & & 0.938 \\
\hline Minimal & $13(65.0)$ & $14(70.0)$ & \\
\hline Moderate & $6(30.0)$ & $5(25.0)$ & \\
\hline Severe & $1(5.0)$ & $1(5.0)$ & \\
\hline Final pathology & & & 0.784 \\
\hline Dysplasia & $7(35.0)$ & $9(45.0)$ & \\
\hline EGC & $13(65.0)$ & $11(55.0)$ & \\
\hline Differentiated cancer & 20 (100) & $20(100)$ & - \\
\hline Submucosal invasion & $2(10.0)$ & $1(5.0)$ & 0.999 \\
\hline
\end{tabular}

Date are presented as mean \pm SD or number $(\%)$.

BMI, body mass index; EGC, early gastric cancer.

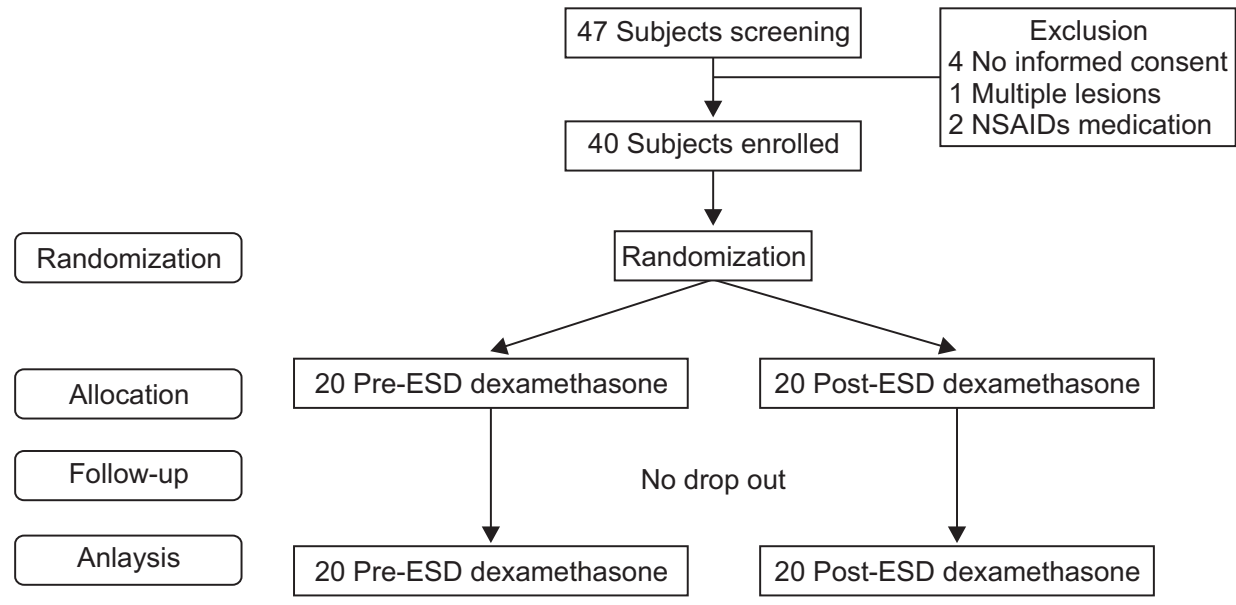

Fig. 1. Study flow. Initially, 47 patients were scheduled to undergo endoscopic submucosal dissection (ESD) for a gastric adenoma or cancer. Seven patients were excluded because of the lack of informed consent $(n=4)$, the presence of multiple lesions $(n=1)$, and the use of nonsteroidal antiinflammatory drugs (NSAIDs) $(n=2)$. A total of 40 patients were randomized into two groups. There were no drop outs. 


\section{Primary and secondary outcomes}

We observed a significant reduction in 6-hour PPI in the pre group compared with the post group. $(2.1 \pm 0.8$ vs $3.0 \pm 1.1$, respectively; $p=0.006$ ) (Fig. 2). Immediate PPI was also significantly lower in the pre group than in the post group $(1.6 \pm 0.6$ vs $2.9 \pm 0.6$, respectively; $\mathrm{p}<0.001$ ) (Fig. 2). On post hoc analysis with Bonferroni correction, PPI values were significantly lower in the pre group than in the post group at immediately and 6-hour postoperative. However, there were no differences between the two groups in 12-hour and 24-hour postoperative PPI scores.

Significantly reduced total SF-MP scores at 6 hours were ob-

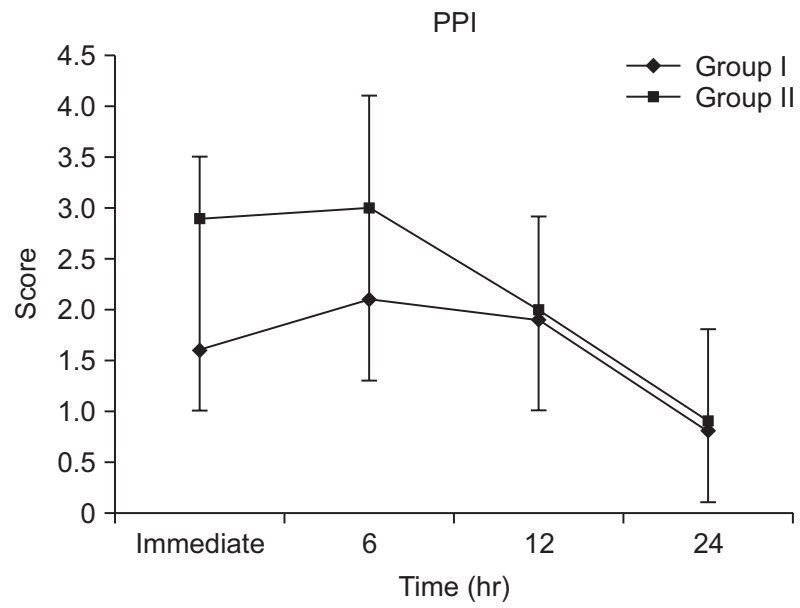

Fig. 2. The trend in present pain intensity (PPI) scores based on the time of administration. The immediate and 6-hour PPI scores were significantly reduced in group I compared with group II. Group I, preendoscopic submucosal dissection (ESD) dexamethasone and post-ESD placebo; Group II, pre-ESD placebo and post-ESD dexamethasone. served in the pre group but not in the post group (12.6 \pm 3.7 vs $15.0 \pm 3.0$, respectively; $p=0.031$ ) (Fig. $3 \mathrm{~A}$ ). Postoperative pain in the sensory domain at 6 hour was particularly lower in the pre group than in the post group $(10.4 \pm 3.7$ vs $12.7 \pm 2.5$, respectively; $p=0.028$ ) (Fig. 3B). The immediate total (pre, $4.4 \pm 1.5$ vs post, $5.8 \pm 2.1 ; \mathrm{p}=0.026)$ and sensory $(2.8 \pm 1.5$ vs $4.4 \pm 2.0$, respectively; $\mathrm{p}=0.006) \mathrm{SF}-\mathrm{MP}$ domain scores were also significantly lower in the pre group than in the post group (Fig. 3B). However, there was no difference in the affective SF-MP scores.

Additional need for the painkiller did not differ between groups (pre, $45.0 \%$ vs post, $65.0 \%$; $p=0.204$ ). For bleeding complications, there was no evidence of bleeding in the pre group patients and only one case (5.0\%) in the post group, which was successfully treated (Table 2).

\section{Multivariate analysis for predictive factors}

We compared the clinical variables between the low $(<3)$ and high $(\geq 3)$ 6-hour PPI groups (Table 3). The low group had significantly more patients from the dexamethasone protocol who were in the pre group and had longer procedure times. To investigate the factors that affected the 6-hour PPI score, we performed univariate and multivariate linear regression analyses (Table 4). Dexamethasone protocol, age, sex, BMI, tumor loca-

Table 2. The Clinical Outcomes after Early Gastric Cancer

\begin{tabular}{lccc}
\hline & $\begin{array}{c}\text { Pre group } \\
(\mathrm{n}=20)\end{array}$ & $\begin{array}{c}\text { Post group } \\
(\mathrm{n}=20)\end{array}$ & p-value \\
\hline En bloc resection & $20(100)$ & $19(95.0)$ & 0.311 \\
Complete resection & $20(100)$ & $19(95.0)$ & 0.311 \\
Complication (bleeding) & 0 & $1(5.0)$ & 0.311 \\
Additional need of painkiller & $9(45.0)$ & $13(65.0)$ & 0.204
\end{tabular}

Data are presented as number (\%).
A

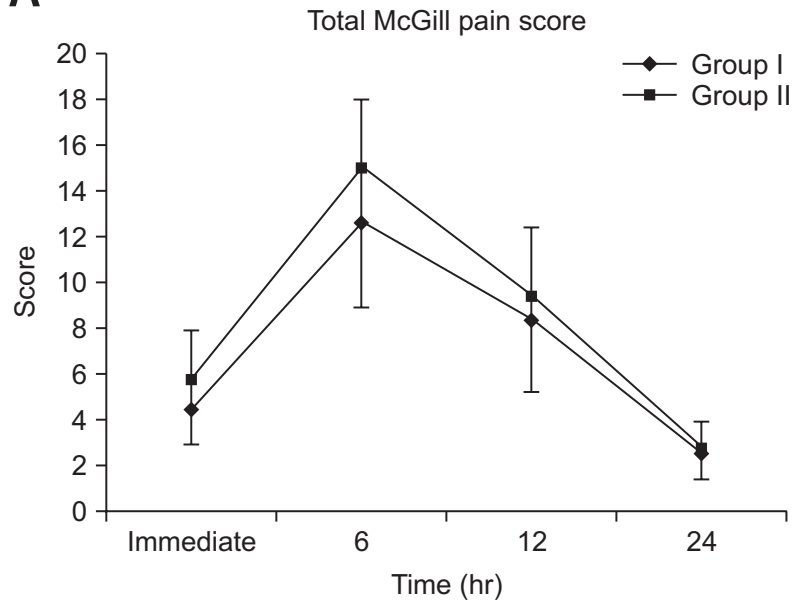

B

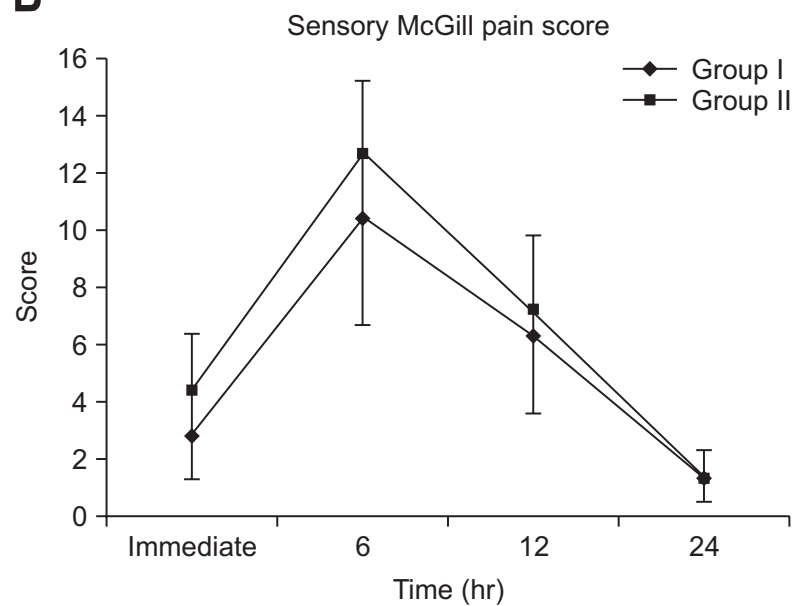

Fig. 3. Trend of the short-form McGill pain (SF-MP) score. (A) Total SF-MP score. The immediate and 6-hour total SF-MP scores were lower in group I than in group II. (B) Sensory SF-MP score. The immediate and 6-hour sensory SF-MP scores were lower in group I than in group II. Group I, pre-endoscopic submucosal dissection (ESD) dexamethasone and post-ESD placebo; Group II, pre-ESD placebo and post-ESD dexamethasone. 
Table 3. Baseline Characteristics of the Low 6 Hour-PPI $(<3)$ and High 6 Hour-PPI $(\geq 3)$ Groups

\begin{tabular}{|c|c|c|c|}
\hline Variable & $\begin{array}{l}\text { Low } 6 \text { hour-PPI } \\
\text { group }(n=21)\end{array}$ & $\begin{array}{l}\text { High } 6 \text { hour-PPI } \\
\text { group }(\mathrm{n}=19)\end{array}$ & p-value \\
\hline Dexamethasone group & & & 0.004 \\
\hline Pre group & 15 (71.4) & $5(26.3)$ & \\
\hline Post group & $6(28.6)$ & $14(73.7)$ & \\
\hline Age, yr & $54.8 \pm 8.2$ & $52.4 \pm 9.3$ & 0.392 \\
\hline BMI, kg/m² & $22.1 \pm 2.1$ & $22.4 \pm 1.6$ & 0.585 \\
\hline Size, mm & $1,628.8 \pm 428.4$ & $1,818.1 \pm 550.6$ & 0.230 \\
\hline Location & & & 0.425 \\
\hline Upper & $5(23.8)$ & 6 (31.6) & \\
\hline Middle & $4(19.0)$ & $6(31.6)$ & \\
\hline Lower & $12(57.1)$ & $7(36.8)$ & \\
\hline Procedure time, min & $48.0 \pm 12.0$ & $38.5 \pm 9.6$ & 0.010 \\
\hline Bleeding & & & 0.356 \\
\hline Minimal & 13 (61.9) & $9(47.4)$ & \\
\hline Moderate & $8(38.1)$ & 10 (52.6) & \\
\hline Severe & 0 & 0 & \\
\hline Fibrosis & & & 0.362 \\
\hline Minimal & 13 (61.9) & $14(73.7)$ & \\
\hline Moderate & $6(28.6)$ & $5(26.3)$ & \\
\hline Severe & $2(9.5)$ & 0 & \\
\hline Submucosal invasion & $2(9.5)$ & $1(5.3)$ & 0.609 \\
\hline
\end{tabular}

Data are presented as number (\%) or mean \pm SD.

PPI, present pain intensity; BMI, body mass index.

tion, tumor size, procedure time, severity of bleeding, degree of fibrosis, and submucosa invasion were included in the univariate analyses. Among these, dexamethasone protocol (odds ratio [OR], 7.000; 95\% confidence interval [CI], 1.739 to 28.174; $\mathrm{p}=0.006$ ) and procedure time (OR, 0.908; 95\% CI, 0.837 to 0.986 ; $\mathrm{p}=0.022$ ) were identified as significant factors for lowering the 6-hour PPI score after ESD. Dexamethasone administered before ESD and longer procedure times were associated with lower 6-hour PPI scores. Multivariate analysis identified dexamethasone protocol (OR, 7.474; 95\% CI, 1.586 to $35.221 ; \mathrm{p}=0.011$ ) and procedure time (OR, 0.904; 95\% CI, 0.824 to $0.992 ; \mathrm{p}=0.032$ ) as independent factors for predicting the development of pain after ESD.

\section{DISCUSSION}

Although dexamethasone was reported to be effective in preventing epigastric pain associated with $\mathrm{ESD},{ }^{5}$ the optimal administration timing has not been previously studied. This study demonstrated that dexamethasone administered immediately before ESD had an effective analgesic effect at the immediate and 6-hour postoperative periods compared with administering it after the procedure. Because maximum pain was observed in patients at 6 hours after ESD in our pilot study, a prophylactic analgesic would have had to be effective during this period. Furthermore, this study suggested that preoperative dexamethasone could improve post-ESD pain not only immediately but 6 hours postoperatively, which was not shown in our previous trial with post-ESD dexamethasone administration. ${ }^{6}$

Preemptive analgesia is initiated preoperatively in order to prevent or reduce the memory of the nociceptive stimuli in the central nervous system in order to reduce the need for postoperative analgesics; it has the potential to be more effective than postoperative analgesia. ${ }^{12-15}$ Based on this concept, dexamethasone can also have a preemptive analgesic effect on pain after ESD for early gastric neoplasm. Dexamethasone is used in certain surgical procedures based on its established efficacy and potential analgesic benefits. ${ }^{16-19,22}$ The mechanisms of how glucocorticoids reduce pain are not fully understood but are possibly associated with their anti-inflammatory actions, including suppressing bradykinin ${ }^{23}$ and neuropeptide ${ }^{24}$ and blocking the cyclooxygenase and lipooxygenase pathways and, therefore, prostaglandin production. ${ }^{25,26}$ The onset of glucocorticoid action is generally 1 to 2 hours depending on the administration route because the effects are mostly mediated through protein synthesis $;^{25}$ moreover, early mediators activate immediately after the surgical procedure. Therefore, the timing of glucocorticoid administration is important because it could play the key role in reducing the inflammation that starts immediately after ESD. Glucocorticoid administration later than 1 to 2 hours after ESD might be too late for optimal anti-inflammatory effects.

Concerns have been raised regarding the possible side effects of glucocorticoids. Although dexamethasone generally has a number of adverse effects, ${ }^{26}$ a meta-analysis with data from more than 1,900 surgical patients indicated that perioperative administration of high-dose methylprednisolone was not associated with adverse events. ${ }^{27}$ Our previous study on single-dose postoperative dexamethasone also indicated that there were no serious adverse events associated with healing of the residual ulcer and no bleeding, infection, or increase in blood glucose level. ${ }^{5}$ In this study, there was only one complication, minor bleeding from a post-ESD ulcer.

Our findings confirmed the results of our previous trial on the efficacy of single-dose dexamethasone for pain relief after ESD and also further investigated the effect of the timing of dexamethasone administration as preemptive analgesia, which had not been examined before. Previous studies focused on controlling postoperative pain rather than on preemptive analgesia. ${ }^{2,5-7}$ Pharmacological interventions with local lidocaine, bupivacaine, and transdermal fentanyl patch have demonstrated pain relief after $\mathrm{ESD},{ }^{2,6,7}$ but these also target existing pain. In contrast, our study with dexamethasone administered before ESD involved inducing and maintaining central hypersensitivity. Moreover, our paper included sensitivity analyses such as SF-MP scores for the valid and reliable assessment of the sensory and affec- 
Table 4. Univariate and Multivariate Analysis

\begin{tabular}{|c|c|c|c|c|}
\hline \multirow{2}{*}{ Variable } & \multicolumn{2}{|c|}{ Univariate } & \multicolumn{2}{|c|}{ Multivariate } \\
\hline & OR $(95 \% \mathrm{CI})$ & $\mathrm{p}$-value & OR (95\% CI) & p-value \\
\hline Dexamethasone group & & 0.006 & & 0.011 \\
\hline Pre group & 1 & & 1 & \\
\hline Post group & 7.000 (1.739-28.174) & & $7.474(1.586-35.221)$ & \\
\hline Age & $0.968(0.899-1.042)$ & 0.382 & & \\
\hline Sex & & 0.492 & & \\
\hline Male & 1 & & & \\
\hline Female & $0.642(0.181-2.275)$ & & & \\
\hline BMI & $1.103(0.783-1.555)$ & 0.574 & & \\
\hline \multicolumn{5}{|l|}{ Location } \\
\hline Upper & 1 & & & \\
\hline Middle & $1.250(0.221-7.084)$ & 0.801 & & \\
\hline Lower & $0.486(0.107-2.199)$ & 0.349 & & \\
\hline Specimen size & $1.001(0.999-1.002)$ & 0.232 & & \\
\hline Procedure time & $0.908(0.837-0.986)$ & 0.022 & $0.904(0.824-0.992)$ & 0.032 \\
\hline \multicolumn{5}{|l|}{ Severity of bleeding } \\
\hline Mild & 1 & & & \\
\hline Moderate & $1.806(0.512-6.363)$ & 0.358 & & \\
\hline \multicolumn{5}{|l|}{ Fibrosis } \\
\hline Mild & 1 & & & \\
\hline Moderate & $0.774(0.190-3.159)$ & 0.721 & & \\
\hline Severe & - & - & & \\
\hline \multicolumn{5}{|l|}{ Submucosal invasion } \\
\hline No & 1 & & & \\
\hline Yes & $0.528(0.044-6.337)$ & 0.614 & & \\
\hline
\end{tabular}

OR, odds ratio; CI, confidence interval; BMI, body mass index.

tive aspects of pain. ${ }^{28}$ In our study, univariate and multivariate analyses identified dexamethasone protocol and procedure time as independent factors for predicting the development of pain after ESD. In general, parameters such as the size or location of the tumor are considered predictors of postoperative pain. However in our study, there were no significant differences in these parameters, whereas, unexpectedly, longer procedure time was associated with less postoperative pain. This may imply that delicate dissection of the tumor with minimal submucosal injury reduces postoperative pain even though it takes longer time. Above all, this study shows that pain management is more effective in controlling postoperative pain than any other clinical parameters (Table 3).

Our study had some limitations. The biological mechanisms of preoperative dexamethasone's analgesic effects were not fully clarified. Although we suggested a few theories and clinical evidence to support the role of dexamethasone in preoperative analgesia for ESD, future analyses of biochemical markers are needed to confirm the hypothesis. In addition, the doseresponse relationship and the optimal dosage of dexamethasone require further study because of the potential side effects of high-dose glucocorticoids. Furthermore, because the duration of anti-inflammatory potency varies among the different types of glucocorticoids, the most effective type should be assessed based on mean and maximum post-ESD pain duration. Finally, side effects such as wound healing and hyperglycemia need additional evaluation.

In conclusion, patients who underwent ESD for early gastric neoplasm had more effective pain reduction with preoperative rather than postprocedure dexamethasone administration.

\section{CONFLICTS OF INTEREST}

No potential conflict of interest relevant to this article was reported.

\section{REFERENCES}

1. Oka S, Tanaka S, Kaneko I, et al. Advantage of endoscopic submucosal dissection compared with EMR for early gastric cancer. 
Gastrointest Endosc 2006;64:877-883.

2. Kim B, Lee H, Chung H, et al. The efficacy of topical bupivacaine and triamcinolone acetonide injection in the relief of pain after endoscopic submucosal dissection for gastric neoplasia: a randomized double-blind, placebo-controlled trial. Surg Endosc 2015;29:714-722.

3. Lee $\mathrm{H}$, Cheoi KS, Chung $\mathrm{H}$, et al. Clinical features and predictive factors of coagulation syndrome after endoscopic submucosal dissection for early gastric neoplasm. Gastric Cancer 2012;15:83-90.

4. Onogi F, Araki H, Ibuka T, et al. "Transmural air leak": a computed tomographic finding following endoscopic submucosal dissection of gastric tumors. Endoscopy 2010;42:441-447.

5. Lee HW, Lee H, Chung $\mathrm{H}$, et al. The efficacy of single-dose postoperative intravenous dexamethasone for pain relief after endoscopic submucosal dissection for gastric neoplasm. Surg Endosc 2014;28:2334-2341.

6. Kiriyama S, Oda I, Nishimoto F, Mashimo Y, Ikehara H, Gotoda T. Pilot study to assess the safety of local lidocaine injections during endoscopic submucosal dissection for early gastric cancer. Gastric Cancer 2009;12:142-147.

7. Choi HS, Kim KO, Chun HJ, et al. The efficacy of transdermal fentanyl for pain relief after endoscopic submucosal dissection: a prospective, randomised controlled trial. Dig Liver Dis 2012;44:925929.

8. Baxendale BR, Vater M, Lavery KM. Dexamethasone reduces pain and swelling following extraction of third molar teeth. Anaesthesia 1993;48:961-964.

9. Waldron NH, Jones CA, Gan TJ, Allen TK, Habib AS. Impact of perioperative dexamethasone on postoperative analgesia and side-effects: systematic review and meta-analysis. Br J Anaesth 2013;110:191-200.

10. Diakos EA, Gallos ID, El-Shunnar S, Clarke M, Kazi R, Mehanna $\mathrm{H}$. Dexamethasone reduces pain, vomiting and overall complications following tonsillectomy in adults: a systematic review and meta-analysis of randomized controlled trials. Clin Otolaryngol 2011;36:531-542.

11. De Oliveira GS Jr, Almeida MD, Benzon HT, McCarthy RJ. Perioperative single dose systemic dexamethasone for postoperative pain: a meta-analysis of randomized controlled trials. Anesthesiology 2011;115:575-588.

12. Dahl JB, Møiniche S. Pre-emptive analgesia. Br Med Bull 2004;71:13-27.

13. Homer JJ, Swallow J, Semple P. Audit of pain management at home following tonsillectomy in children. J Laryngol Otol 2001; 115:205-208.

14. Kedek A, Derbent A, Uyar M, et al. Pre-emptive effects of ibuprofen syrup and lidocaine infiltration on post-operative analgesia in children undergoing adenotonsillectomy. J Int Med Res 2005;33:188-195.

15. Wu CT, Yeh CC, Yu JC, et al. Pre-incisional epidural ketamine, morphine and bupivacaine combined with epidural and general anaesthesia provides pre-emptive analgesia for upper abdominal surgery. Acta Anaesthesiol Scand 2000;44:63-68.

16. Bernardo WM, Aires FT. Efficacy of dexamethasone in the prophylaxis of nausea and vomiting during the postoperative period of laparoscopic cholecystectomy. Rev Assoc Med Bras 2013;59:387391.

17. Bianchin A, De Luca A, Caminiti A. Postoperative vomiting reduction after laparoscopic cholecystectomy with single dose of dexamethasone. Minerva Anestesiol 2007;73:343-346.

18. Bilgin TE, Birbicer H, Ozer Z, Doruk N, Tok E, Oral U. A comparative study of the antiemetic efficacy of dexamethasone, ondansetron, and metoclopramide in patients undergoing gynecological surgery. Med Sci Monit 2010;16:CR336-CR341.

19. Feroci F, Rettori M, Borrelli A, Lenzi E, Ottaviano A, Scatizzi M. Dexamethasone prophylaxis before thyroidectomy to reduce postoperative nausea, pain, and vocal dysfunction: a randomized clinical controlled trial. Head Neck 2011;33:840-846.

20. Lee H, Yun WK, Min BH, et al. A feasibility study on the expanded indication for endoscopic submucosal dissection of early gastric cancer. Surg Endosc 2011;25:1985-1993.

21. Hawker GA, Mian S, Kendzerska T, French M. Measures of adult pain: Visual Analog Scale for Pain (VAS Pain), Numeric Rating Scale for Pain (NRS Pain), McGill Pain Questionnaire (MPQ), Short-Form McGill Pain Questionnaire (SF-MPQ), Chronic Pain Grade Scale (CPGS), Short Form-36 Bodily Pain Scale (SF-36 BPS), and Measure of Intermittent and Constant Osteoarthritis Pain (ICOAP). Arthritis Care Res (Hoboken) 2011;63 Suppl 11:S240S252.

22. Lee Y, Lai HY, Lin PC, Lin YS, Huang SJ, Shyr MH. A dose ranging study of dexamethasone for preventing patient-controlled analgesia-related nausea and vomiting: a comparison of droperidol with saline. Anesth Analg 2004;98:1066-1071.

23. Hargreaves KM, Costello A. Glucocorticoids suppress levels of immunoreactive bradykinin in inflamed tissue as evaluated by microdialysis probes. Clin Pharmacol Ther 1990;48:168-178.

24. Hong D, Byers MR, Oswald RJ. Dexamethasone treatment reduces sensory neuropeptides and nerve sprouting reactions in injured teeth. Pain 1993;55:171-181.

25. Sapolsky RM, Romero LM, Munck AU. How do glucocorticoids influence stress responses? Integrating permissive, suppressive, stimulatory, and preparative actions. Endocr Rev 2000;21:55-89.

26. Brunton LL, Chabner BA, Knollmann BC. Goodman and Gilman's the pharmacological basis of therapeutics. 12th ed. New York: McGraw Hill Medical, 2011.

27. Sauerland S, Nagelschmidt M, Mallmann P, Neugebauer EA. Risks and benefits of preoperative high dose methylprednisolone in surgical patients: a systematic review. Drug Saf 2000;23:449-461.

28. Breivik H, Borchgrevink PC, Allen SM, et al. Assessment of pain. Br J Anaesth 2008;101:17-24. 